\title{
MODELAGEM MATEMÁTICA DE UM PROCESSO DE CONSUMO: O CASO ILUSTRATIVO DE UMA VISTORIA VEICULAR
}

\author{
Eduardo Galvão Moura Jardim \\ Universidade Federal do Rio de Janeiro \\ Departamento de Engenharia Industrial - UFRJ \\ eduardojardim@poli.ufrj.br \\ Leonardo Aragão Guimarães \\ Universidade do Grande Rio José Souza Herdy - UNIGRANRIO \\ Escola de Ciências e Tecnologia - ECT \\ professorleo@unigranrio.edu.br \\ Marcos Vinícius dos Santos Romualdo \\ Universidade Federal do Rio de Janeiro \\ Departamento de Engenharia Industrial - UFRJ \\ mvinicius7@poli.ufrj.br
}

\begin{abstract}
RESUMO
O presente trabalho propõe a análise quantitativa da jornada de consumo presente na prestação do serviço público através de um modelo matemático de consumo lean, tomando como estudo de caso o processo de vistoria veicular do DETRAN RJ. Diante dos dados gerados pelo modelo, o trabalho foi direcionado à proposição de melhorias. $\mathrm{O}$ estudo de campo mostrou uma jornada de 2 h 07 min para a conclusão do procedimento. Com base nas premissas preconizadas pelo pensamento lean e conceitos de valor e desperdício, propôsse uma reformulação capaz de oferecer potenciais melhorias à experiencia do consumidor. Os resultados obtidos sugerem uma redução de $65 \%$ das etapas do processo, de $95 \%$ do desperdício gerado e um aumento de $54 \%$ no valor agregado ao consumidor. Tais valores evidenciam como tal abordagem pode ser útil para a melhoria de processos de consumo em geral.
\end{abstract}

Palavra-chave: Consumo Lean, Vistoria Veicular, Mapas de Fluxo De Valor.

\begin{abstract}
The present work proposes the quantitative analysis of the consumption journey present in the public service rendering through a mathematical model of lean consumption, taking as a case study the DETRAN RJ vehicular inspection process. Given the data generated by the model, the work was directed to the proposition of improvements. The field study showed a $2 \mathrm{~h} 07$ min journey to complete the procedure. Based on the assumptions advocated by lean thinking and concepts of value and waste, a reformulation was proposed to offer potential improvements to the consumer experience. The results suggest a $65 \%$ reduction in the process steps, a 95\% reduction in waste generated and a 54\% increase in consumer value added. These values show how such an approach can be useful for improving overall consumer processes.
\end{abstract}

Keywords: Lean Consumption, Vehicular Survey, Value Stream Maps. 


\section{Como Citar:}

JARDIM, Eduardo Galvão Moura; GUIMARÃES, Leonardo Aragão; ROMUALDO, Marcos Vinícius dos Santos. Modelagem Matemática De Um Processo De Consumo: O Caso Ilustrativo De Uma Vistoria Veicular. In: SIMPÓSIO DE PESQUISA OPERACIONAL E LOGÍSTICA DA MARINHA, 19., 2019, Rio de Janeiro, RJ. Anais [...]. Rio de Janeiro: Centro de Análises de Sistemas Navais, 2019.

\section{INTRODUÇÃO}

Ao se pensar sobre a evolução da sociedade moderna, e mais especificamente sobre as Organizações, conclui-se que estas foram - e são criadas - com um propósito em comum: atender às necessidades de seus clientes, sobretudo nos aspectos ligados ao tempo, qualidade e preço. Nesse sentido, num breve resumo histórico, tivemos primeiro a mecanização da manufatura, centrada primeiramente no tear e máquina a vapor. Em seguida conseguimos integrar as operações de produção pela eletricidade, viabilizando a linha de montagem. Depois, com o advento da eletrônica, chegamos ao computador e aos primeiros processos automatizados. Em continuidade a esses três grandes marcos tecnológicos - mecanização, eletricidade e eletrônica - chegamos hoje a $4^{\mathrm{a}}$. Revolução Industrial, caracterizada também como Indústria 4.0. Agora, num mundo que vai se digitalizando por completo, "tudo" e "todos” ficam cada vez mais conectados através da Tecnologia Informação.

Dispondo assim de um vasto arsenal de informações operados por algoritmos de inteligência artificial, processos automatizados são também capazes de - eficiente e eficazmente - configurar e fazer produtos de acordo com características ou necessidades individuais de diferentes clientes. O avanço e consolidação desse tipo de produção pode, em futuro não tão distante, significar o fim da produção em massa como hoje conhecemos. Nesse contexto, em uso, três recursos de produção já são bem conhecidos: as Impressoras 3D, os Drones e os Veículos Autômatos. Nessa nova era, assim como os processos, produtos também se tornam inteligentes. Com chips proativos neles embutidos, vão sendo exclusivamente identificáveis. Podem ser localizados em todos os momentos. Conhecem o seu próprio histórico, status atual e rotas alternativas para alcançar seu estado de destino que pode incluir inclusive a sua reciclagem.

Em suma, as empresas, de uma forma geral passaram anos de sua existência direcionando seus esforços no desenvolvimento de processos, produtos e serviços cada vez melhores. Contudo pode-se dizer que não dedicaram igual prioridade a entender e aprimorar a jornada ou o processo de consumo do cliente. Muitas delas oferecem excelentes produtos, bens e serviços, mas impõem jornadas de consumo imersas em filas, esperas, falta de suporte técnico e até mesmo dificuldades de pagamentos, imprimindo más experiencias aos consumidores. O problema se agrava ainda mais quando os olhares são voltados para o setor público, onde a falta de competitividade e até mesmo a permanecia das organizações independe da satisfação dos cidadãos. Logo, questiona-se como será possível atender aos requisitos dos clientes de prazo, tempo e qualidade e, além disso, mitigar os desperdícios e custos associados dos provedores de serviço - sejam eles privados ou públicos - de forma a garantir a competência esperada.

Dentre diversas formas do estudo e análise utilizados como ferramentas de melhoria na jornada de consumo do cliente, a modelagem matemática se mostra uma importante ferramenta utilizada como suporte para a tomada de decisão em processos de produção, logística, expansão, investimentos e outros. Com base nos fundamentos estabelecidos por Womack e Jones (2006), Jardim (2014) propôs um modelo matemático que busca definir, classificar e quantificar diferentes formas de desperdício e valor presentes em uma jornada de consumo, transformando os dados em informações úteis para a avaliação do processo e 
proposição de melhorias.

\section{OBJETIVO}

O presente artigo se propõe a avaliar quantitativamente uma jornada de consumo de um serviço prestado pelo setor público brasileiro através da aplicação do modelo matemático de consumo lean proposto por Jardim (2014). Espera-se com isso eliminar etapas do processo que não agreguem valor ao consumidor, melhorar sua experiência de consumo como um todo e, consequentemente, gerar insumos para a proposição de melhorias do processo.

\section{METODOLOGIA}

Para fins de metodologia, este trabalho é balizado por uma abordagem empreendedora-tecnológica, valorizando-se a profundidade em detrimento da amplitude.

Esta abordagem, pauta sua causa e origem no reconhecimento de um problema, ineficiência, anseio ou necessidade massiva do meio social (potencial mercado consumidor) que careça de resolução ou suprimento. Dessa forma, são identificadas oportunidades no desenvolvimento de produtos e serviços que possam ser efetivamente transformadas em negócios geradores de riqueza e bem-estar social.

Valorizar a profundidade em detrimento da amplitude significa dizer que, de forma geral, será necessário estudar uma situação em um nicho específico de forma mais detalhada possível, ao invés de expandir os horizontes de forma mais genérica. Com isso, espera-se obter resultados mais concretos que possam ser aplicados e extrapolados para outras situações.

\section{FUNDAMENTAÇÃO TEÓRICA}

O cenário competitivo no qual as empresas estão inseridas, aliada a uma preocupação atual com a questão dos desperdícios, tem exigido um tratamento rápido e dinâmico das informações. Neste contexto, a filosofia lean tem se mostrado uma relevante ferramenta na busca de vantagens competitivas que representem um relacionamento mais estreito entre provedores e clientes.

De acordo com Costa \& Jardim (2015), o pensamento enxuto é uma maneira de pensar uma melhor organização de um ambiente produtivo. A aposta-chave é que, ao entender o que é valor para o cliente, sejamos capazes de identificar e eliminar os desperdícios através do melhoramento contínuo dos processos, e assim alavancar a sua posição competitiva.

Logo, espera-se que a filosofia lean, identificando o que é valor para o cliente, possa contribuir para a mitigação de desperdícios, um melhor controle sobre a qualidade, e no aperfeiçoamento do desempenho dos processos. (GUIMARÃES \& GUIMARÃES, 2016)

\section{- 4.1. CONSUMO LEAN}

Womack \& Jones (2006), formulam o conceito de “Consumo Lean” voltando seu olhar para o processo de consumo, identificando o que é valor para o consumidor e o que é desperdício, procurando assim eliminá-lo. Este é fundamentado em seis princípios básicos que expressam o desejo do consumidor e consequentemente define o que é valor para ele, tais princípios figuram da seguinte forma:

a) Resolva o problema do cliente completamente;

b) Não desperdice o tempo do cliente;

c) Forneça exatamente o que o cliente deseja; 
d) Entregue o valor onde o cliente deseja;

e) Proporcione valor quando o cliente desejar;

f) Reduza a quantidade de decisões que o cliente tem de tomar para resolver seu problema.

- 4.2. MODELO MATEMÁtico PARA AVALIAÇÃo QUANTITATIVA DE UMA JORNADA DE CONSUMO

As seções seguintes se dedicam a apresentar o modelo matemático proposto por Jardim (2014), explicando suas premissas, variáveis e indicadores.

\subsubsection{Premissas do modelo:}

- Todo o processo de consumo - que consome o nosso tempo - possui dois tipos de etapas que podem gerar Valor (V) ou Desperdício (D) para o cliente, sendo estas: Etapas fins (geradoras de valor) e Etapas Associadas (geradoras de desperdício).

- Uma etapa fim é definida como aquela que está estritamente ligada a razão de ser do consumo. Uma etapa associada, por sua vez, não representa a razão de ser do consumo, mas está presente, algumas vezes de forma inevitável, devido ao modelo de processo estabelecido.

\subsubsection{Variáveis do Modelo:}

A partir de tais premissas são definidas variáveis para o modelo, considerando etapas fim e etapas associadas.

\section{Para as etapas fim temos:}

- $\mathbf{T e f ( i )}$ é o tempo da etapa fim (i) medido in loco para $\mathrm{i}=1,2, \ldots$, n etapas fins

- Vef(i) é o tempo de valor da etapa fim (i) para i = 1, 2, .., n etapas fins. Esse tempo é um dado de entrada para o modelamento do fluxo de valor do consumo. Deve ser o tempo padrão ou o tempo racional para realizar uma dada atividade fim (i). Pode resultar de análises de benchmarking, ou de padrões medidos pelo provedor ou pela expectativa do cliente.

- Def(i) é o tempo de desperdício de uma etapa fim para i = 1, 2, .., n etapas fins.

$$
\text { Def(i) = Tef(i) - Vef(i). }
$$

Esse tempo é um dado de saída para o modelamento do fluxo de valor do consumo. Ou seja, se uma atividade fim durar mais que o tempo padrão, esse tempo será considerado desperdício.

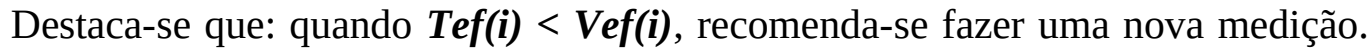
Caso isso se repita na nova medição, é sinal de que o valor padrão dessa etapa i não está coerente. Logo, deve-se rever o que foi considerado como padrão para a etapa i 
Para as etapas associadas temos:

- $\mathbf{T e a}(\boldsymbol{j})$ é o tempo da etapa associada (j) medido in loco para $\mathrm{j}=1,2, \ldots$, m etapas associadas;

- $\boldsymbol{V e a}(\boldsymbol{c}, \mathbf{j})$ é o tempo de valor complementar da etapa associada $(\mathrm{j})$ para $\mathrm{i}=1$, $2, \ldots, \mathrm{m}$ etapas associadas.

- $\boldsymbol{V e a}(\mathbf{s}, \mathbf{j})$ é o tempo de valor subsidiário da etapa associada (j) para i $=1$, 2, ...,m etapas associadas.

- Dea(j) é o tempo de desperdício absoluto de uma etapa associada para j = 1, 2, ..., m etapas associadas.

$$
\operatorname{Dea}(j)=\mathrm{Tea}(j)-[\mathrm{Vea}(c, j)+\mathrm{Vea}(s, j)]
$$

Destaca-se que: Quando $\operatorname{Tef}(j)<[\operatorname{Vea}(c, j)+\operatorname{Vea}(s, j)]$, recomenda-se fazer uma nova medição. Repare também que, em caso de não haver no processo tempos de valores complementares e subsidiários, o tempo todas etapas associadas nada mais é do que desperdício. Os conceitos de etapa de valor complementar e etapa de valor subsidiário são definidos como:

- $\quad$ Etapa de valor complementar: São atividades que auxiliam de alguma forma a atividade fim (razão de ser do consumo). Exemplo: enquanto o cliente se desloca ao restaurante, já pode (através do seu celular) acessar/ler/ouvir o menu dos pratos que poderá vir a escolher;

- Etapas de valor Subsidiário: São atividades que - embora não auxiliem a atividade fim - podem amenizar a sensação de desperdício absoluto. Exemplo: no hall de entrada de um restaurante, enquanto aguarda a disponibilidade de uma mesa para almoçar ou jantar, o cliente pode assistir a um canal de televisão com as últimas notícias do dia.

Apesar de não serem em essência a razão de ser consumo, esses tempos mitigam a sensação de desperdício.

\subsubsection{Indicadores Absolutos do Processo de Consumo}

- $\quad$ - Quantidade de etapas fins;

- $\mathbf{m}$ - Quantidade de etapas associadas;

- $\quad$ ef - Tempo total das etapas fins....................................T ef $=i \sum_{i=1}^{n} T$ ef $(i)$

- $\quad$ T ea - Tempo total das etapas associadas........................T $e a=i \sum_{j=1}^{m} T e a(j)$ 
- $\mathbf{T}$ - Tempo total de duração do processo de consumo. . $\mathrm{T} \dot{T}$ ef $+\mathrm{Tea}$

- $\mathbf{V}$ - Tempo total de valor do consumo..................................... $\mathrm{V} i \sum_{i=1}^{n} V$ ef $(i)$

- $\operatorname{Def}(T)$ - Tempo de desperdício das etapas fins............D ef $(T)=\sum_{i=1}^{n} \operatorname{Def}(\boldsymbol{i})$

- $\quad$ Dea $(T)$ - Tempo de desperdício das etapas associadas....D ea $(T)=\sum_{j=1}^{m} \operatorname{D} e a(j)$

- $\quad D$ - Tempo total de desperdício....................................

- Vc - Tempo total de valor complementar.......................... Vc $i \sum_{j=1}^{m} V e a(c, j)$

- Vs - Tempo total de valor subsidiário................................ Vs $i \sum_{j=1}^{m} V e a(s, j)$

\subsubsection{Indicadores Relativos do Processo de Consumo:}

- $\operatorname{Valor} \operatorname{Real}(\%)=(\mathrm{V} / \mathrm{T}) \times 100$

- $\quad$ "Densidade" das Etapas de Valor $=[\mathrm{n} /(\mathrm{n}+i \mathrm{~m})]$ x 100

- Desperdício Crítico e Urgente $=[\mathrm{Dea}(T) / \mathrm{T}]$ x 100

- Desperdício Emergencial $=[D / T] \times 100$

- Desperdício Total $=\left[\left(\mathrm{Vc}_{\mathrm{c}}+\mathrm{V} \mathrm{Vs}+\mathrm{D} i / \mathrm{T}\right] \mathrm{x} 100=(100-\mathrm{Valor}\right.$ Real $)$

- Grau Atenuante de Desperdício = $\left[\left(\mathrm{Vc}_{\mathrm{c}}+\mathrm{V} \mathrm{Vs} i /(\mathrm{Vc}+i \mathrm{Vs}+D i] \times 100\right.\right.$

- Variabilidade no Atendimento - $\Delta=[(T$ ef $-V i / V i \times 100$

Nota: quão maior for o valor de $\Delta$, pior estão as etapas fins (de valor) do processo em relação ao projetado, configurando um "mau" atendimento; quão menor $\Delta$, melhor estão as etapas fins (de valor) do processo em relação ao projetado. Se $\boldsymbol{\Delta}=\mathbf{0}$, o atendimento (a realidade) está ocorrendo conforme projetado

\section{ESTUDO DE CAMPO}

Dentre muitas opções de órgão públicos e processos de serviço por estes oferecidos escolheu-se o Departamento de Trânsito do Estado do Rio de Janeiro (DETRAN RJ) e o processo de vistoria veicular, como campo de estudo para a proposta do presente trabalho.

O processo de vistoria veicular em particular, deve ser feito anualmente, tendo 
como objetivo garantir que os veículos em trânsito distrital estejam em condições mínimas de segurança e sustentabilidade ambiental, não oferecendo riscos ao próprio condutor ou aos demais cidadãos, nem agredindo de forma excessiva o meio ambiente por suas emissões. Entretanto, o processo sofre com demasiadas ineficiências, como longas filas, atendimentos demorados, dificuldade no agendamento, falhas/lentidão nos sistemas, entre outros. Tais fatores impactam profundamente a qualidade de serviço percebida pelo cliente/cidadão, aumentando com isso seu nível de insatisfação. O procedimento é, inclusive, alvo de intensas discussões sobre sua legitimidade.

O presente trabalho foi pautado por um estudo de campo, representado por uma jornada de consumo real de um processo de vistoria veicular em um posto do DETRAN RJ, a qual foi subdividida em etapas componentes, cada qual classificada como geradora de valor (etapa fim) ou de desperdício (etapa associada) para o consumidor. Adicionalmente foram medidos seus respectivos tempos de processamento. Com os dados em mãos procedeu-se à inserção dos destes no modelo matemático. Finalmente foram feitas a reflexões sobre o processo atual, formulações de propostas de melhorias e avaliação destas.

\subsection{A JORNADA}

A jornada de consumo é iniciada pelo agendamento da vistoria na página do DETRAN RJ, onde são preenchidos dados como placa do veículo, código RENAVAM, CPF/CNPJ do condutor, região de localização, etc. Em seguida, seleciona-se a unidade de vistoria, o turno e o horário para a realização do processo. Uma vez realizado o agendamento, o condutor deve dirigir-se ao posto escolhido, no dia e horário marcado. $\mathrm{O}$ deslocamento realizado teve sua origem no Bairro da Taquara e destino no posto de vistoria da Barra da Tijuca, zona oeste do da cidade do Rio de janeiro, totalizando 14,4 Km.

Chegando ao posto o condutor deve apresentar-se à recepção, onde a sua entrada é registrada e entregue-lhe uma senha, constando a linha (raia) na qual este deve se alocar em fila aos demais veículos e esperar por sua vez de passar pela vistoria. Ao atender o condutor o fiscal solicita-lhe que posicione seu veículo no local por ele indicado, acopla uma sonda no escapamento do veículo para medição das emissões de gases e inicia os registros no sistema (nome do proprietário, placa do veículo, número do chassi, etc.), procedimento aqui denominado set up da vistoria. Em seguida a vistoria é efetivamente iniciada, onde o fiscal avalia visualmente uma série de características estruturais do veículo como carroceria, pneus, espelhos retrovisores, entre outros. Em seguida, solicita ao condutor que siga uma série de comandos (ex.: ligar a seta direita/esquerda, pressionar o freio, ascender faróis) que o possibilitem verificar o funcionamento de um conjunto de itens.

Finalizada a vistoria passa-se à etapa de emissão do laudo, onde o condutor deve permanecer em espera. Sendo o laudo positivo o condutor deve estacionar o veículo em local indicado e dirigir-se ao centro de emissão de CRLV's, onde é retirada uma senha e aguardase pelo atendimento. Uma vez chamada a senha, o cidadão dirige-se ao guichê, entrega o laudo e identidade, assina uma declaração, recebe seu novo CRLV e retira-se do posto, quando então o processo é finalizado. A figura 1 apresenta um esquema gráfico das etapas descritas. 
Figura 1 - Representação esquemática do processo de vistoria e licenciamento anual de veículos

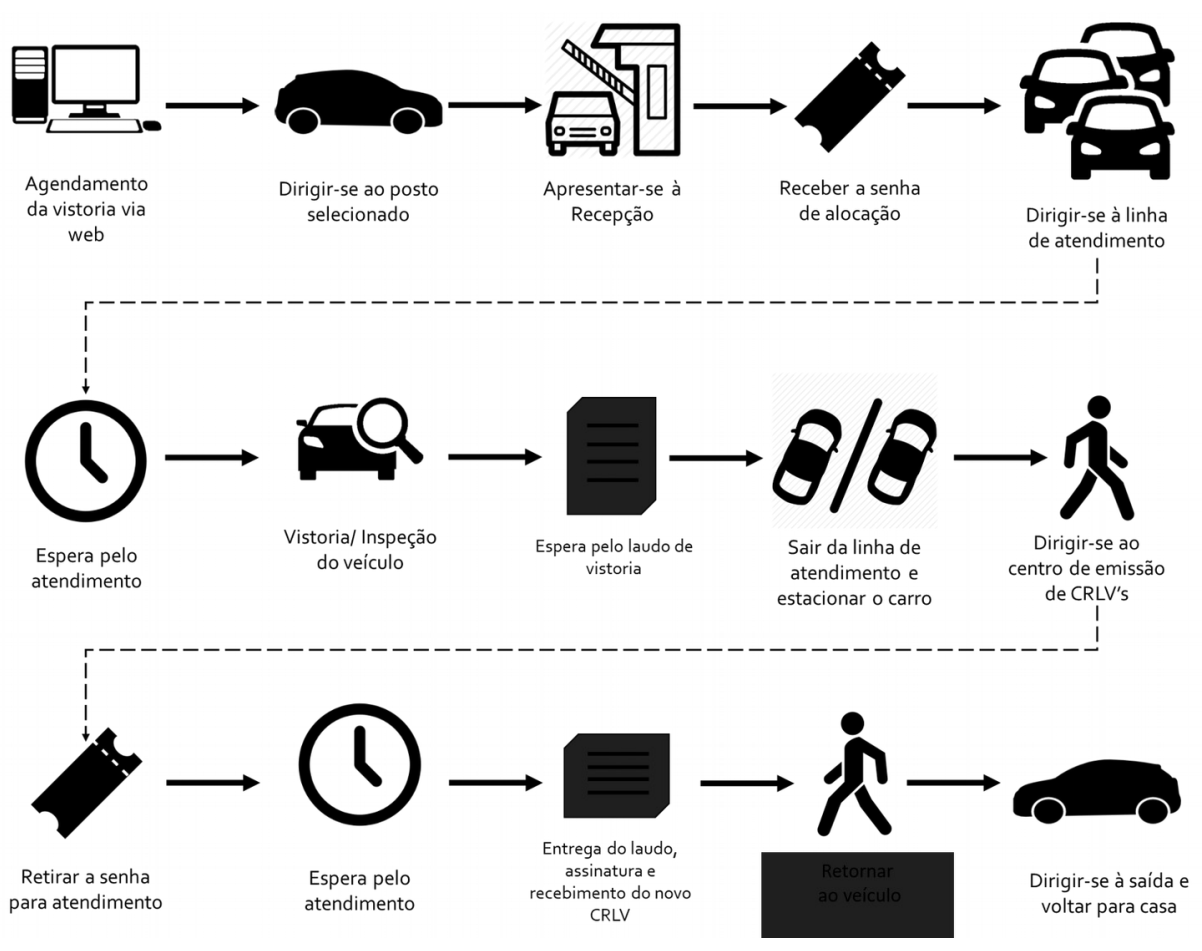

Fonte: Os autores (2018)

Cada etapa foi então classificada como "fim" ou "associada", e teve seus tempos cronometrados a fim de se estruturar o mapa de fluxo de valor representativo da jornada de consumo, como mostra a tabela 1

Tabela 1 - Tempos medidos para o processo de vistoria veicular 


\begin{tabular}{llccc}
\hline \multicolumn{1}{c}{ Etapa } & Classificação & Duração & Duração acumulada \\
\hline 1 & Agendar vistoria & Etapa fim & $00: 03: 20$ & $00: 03: 20$ \\
2 & Dirigir-se ao posto & Etapa Associada & $00: 20: 42$ & $00: 24: 02$ \\
3 & Apresentar-se na recepção & Etapa Associada & $00: 00: 40$ & $00: 24: 42$ \\
4 & Receber Senha & Etapa Associada & $00: 01: 00$ & $00: 25: 42$ \\
5 & Dirigir-se à fila & Etapa Associada & $00: 00: 35$ & $00: 26: 17$ \\
6 & Espera pelo atendimento & Etapa Associada & $00: 06: 15$ & $00: 32: 32$ \\
7 & Set up da vistoria & Etapa Associada & $00: 03: 25$ & $00: 35: 57$ \\
8 & Vistoria & Etapa fím & $00: 02: 54$ & $00: 38: 51$ \\
9 & Emissão do laudo & Etapa Associada & $00: 02: 05$ & $00: 40: 56$ \\
10 & Estacionar o veículo & Etapa Associada & $00: 04: 06$ & $00: 45: 02$ \\
11 & Dirigir-se ao setor de emissão de CRLV & Etapa Associada & $00: 01: 30$ & $00: 46: 32$ \\
12 & Pegar a senha & Etapa Associada & $00: 00: 45$ & $00: 47: 17$ \\
13 & Espera pela chamada & Etapa Associada & $00: 47: 53$ & $01: 35: 10$ \\
14 & Receber novo CRLV & Etapa fim & $00: 03: 28$ & $01: 38: 38$ \\
15 & Dirigir-se ao veículo & Etapa Associada & $00: 01: 41$ & $01: 40: 19$ \\
16 & Dirigir-se à saída & Etapa Associada & $\mathbf{0 0 : 0 1 : 5 3}$ & $01: 42: 12$ \\
17 & Retornar para casa & Etapa Associada & $00: 25: 29$ & $02: 07: 41$ \\
\hline Tempo total do processo & & & 2h 07 min \\
\hline
\end{tabular}

Fonte: Os autores (2018)

Verifica-se na tabela 1 uma grande quantidade de etapas associadas em relação às etapas fins, representando um elevado nível de desperdício ao consumidor.

\section{APLICAÇÃO DO MODELO MATEMÁTICO}

\subsection{DEFINIÇão DOS VALORES PADRÕES - Vef(i)}

Conforme o modelo proposto, faz-se necessário o estabelecimento de valores de tempos-padrões para as etapas fim, de forma a avaliar o processo. Uma vez que não foram encontrados na literatura dados de benchmark para um processo de vistoria veicular foram estabelecidos tempos padrões de acordo com a percepção pessoal dos autores, na condição de consumidor do processo, como apresenta a tabela 2.

Tabela 2 - Tempos padrões para as etapas-fim do processo de vistoria na visão do autor do estudo.

\begin{tabular}{ll}
\hline \multicolumn{2}{c}{ Tempos padrões para as etapas fim } \\
\hline Tempo padrão para o agendamento da vistoria & $2 \mathrm{~min}$ \\
Tempo padrão para a vistoria & $3 \mathrm{~min}$ \\
Tempo padrão para o recebimento do novo CRLV & $2 \mathrm{~min}$ \\
\hline
\end{tabular}

Fonte: Os autores (2018)

\subsection{CÁLCULO DAS VARIÁVEIS E INDICADORES DO PROCESSO}

Aplicando-se no modelo matemático os dados coletados em campo, assim como os tempos padrões definidos anteriormente, foi possível obter um diagnóstico quantitativo da eficiência do processo. A tabela 3 apresenta os tempos de cada etapa, assim como os tempos padrões e desperdícios. As tabelas 4 e 5 apresentam respectivamente as variáveis absolutas e relativas propostas pelo modelo matemático. 
Tabela 3 - Tempos medidos para o processo de vistoria veicular

\begin{tabular}{lcccc}
\hline \multicolumn{1}{c}{ Etapa } & Classificação & $\begin{array}{c}\text { Tempos } \\
\text { Tef(i) / Tea(j) }\end{array}$ & $\begin{array}{c}\text { Tempos Padrões } \\
\text { Tef(i) / Tea(j) }\end{array}$ & $\begin{array}{c}\text { Desperdícios } \\
\text { Def(i) / Dea(j) }\end{array}$ \\
\hline Agendar vistoria & Etapa fim & $0: 03: 20$ & $0: 02: 00$ & $00: 01: 20$ \\
Dirigir-se ao posto & Etapa Assoc. & $0: 20: 42$ & & $00: 20: 42$ \\
Apresentar-se na recepção & Etapa Assoc. & $0: 00: 40$ & & $00: 00: 40$ \\
Receber Senha & Etapa Assoc. & $0: 01: 00$ & & $00: 01: 00$ \\
Dirigir-se à fila & Etapa Assoc. & $0: 00: 35$ & & $00: 00: 35$ \\
Espera pelo atendimento & Etapa Assoc. & $0: 06: 15$ & & $00: 06: 15$ \\
Set up da vistoria & Etapa Assoc. & $0: 03: 25$ & $00: 03: 25$ \\
Vistoria & Etapa fim & $0: 02: 54$ & $0: 03: 00$ & $00: 00: 00$ \\
Emissão do laudo & Etapa Assoc. & $0: 02: 05$ & & $00: 02: 05$ \\
Estacionar o veículo & Etapa Assoc. & $0: 04: 06$ & & $00: 04: 06$ \\
Dirigir-se ao setor de CRLV's & Etapa Assoc. & $0: 01: 30$ & & $00: 01: 30$ \\
Pegar a senha & Etapa Assoc. & $0: 00: 45$ & & $00: 00: 45$ \\
Espera pela chamada & Etapa Assoc. & $0: 47: 53$ & & $00: 47: 53$ \\
Receber novo CRLV & Etapa fim & $0: 03: 28$ & $0: 02: 00$ & $00: 01: 28$ \\
Dirigir-se ao veículo & Etapa Assoc. & $0: 01: 41$ & & $00: 01: 41$ \\
Dirigir-se à saída & Etapa Assoc. & $0: 01: 53$ & & $00: 01: 53$ \\
Retornar para casa & Etapa Assoc. & $0: 25: 29$ & & $00: 25: 29$ \\
\hline \multicolumn{1}{c}{ Tempos Totais } & & $\mathbf{0 2 : 0 7 : 4 1}$ & $\mathbf{0 : 0 7 : 0 0}$ & $\mathbf{0 2 : 0 0 : 4 7}$ \\
\hline
\end{tabular}

Fonte: Os autores (2018)

Vale enfatizar que o modelo estabelece tempos padrões apenas para as etapas fim do processo, e que o desperdício de uma etapa fim se dá a partir da extrapolação deste pelo processo.

Tabela 4 - Indicadores absolutos obtidos para o processo de vistoria veicular

\begin{tabular}{lc}
\hline \multicolumn{1}{c}{ Indicadores absolutos do processo de consumo } & Valor medido \\
\hline Quantidade de etapas fins $(\mathrm{m})$ & 3 \\
Quantidade de etapas associadas $(\mathrm{n})$ & 14 \\
Tempo total de etapas fim $\left(\mathrm{T}_{\mathrm{ef}}\right)$ & $00: 09: 42$ \\
Tempo total de etapas associadas $\left(\mathrm{T}_{\mathrm{ea}}\right)$ & $01: 57: 59$ \\
Tempo total de duração do processo de consumo $(\mathrm{T})$ & $02: 07: 41$ \\
Tempo total de valor do consumo $(\mathrm{V})$ & $00: 06: 54$ \\
Tempo total de desperdício das etapas fim - $\left[\mathrm{D}_{\mathrm{ef}}(\mathrm{T})\right]$ & $00: 02: 48$ \\
Tempo total de desperdício das etapas associadas $-\left[\mathrm{D}_{\mathrm{ea}}(\mathrm{T})\right]$ & $01: 57: 59$ \\
Tempo total de desperdício $(\mathrm{D})$ & $02: 00: 47$ \\
Tempo total de valor complementar $\left(\mathrm{V}_{\mathrm{c}}\right)$ & $00: 00: 00$ \\
Tempo total de valor subsidiário $\left(\mathrm{V}_{\mathrm{s}}\right)$ & $00: 00: 00$ \\
\hline
\end{tabular}

Fonte: Os autores (2018)

Observa-se que no processo de vistoria não houve ocorrências de etapas cujos tempos pudessem ser classificados como geradores de valor complementar ou subsidiário ao consumidor, de forma que tais variáveis foram consideradas nulas nos cálculos.

Tabela 5 - Indicadores relativos obtidos para o processo de vistoria veicular 


\begin{tabular}{lcc}
\hline \multicolumn{1}{c}{ Indicadores relativos do processo de consumo } & Meta & Valor medido \\
\hline Valor real & $100 \%$ & $5 \%$ \\
Densidade de etapas de valor & $100 \%$ & $18 \%$ \\
Desperdício crítico e urgente & $0 \%$ & $92 \%$ \\
Desperdício emergencial & $0 \%$ & $95 \%$ \\
Desperdício total & $0 \%$ & $95 \%$ \\
Grau de atenuidade de desperdício & $100 \% *$ & $0 \%$ \\
Variabilidade do atendimento & $0 \%$ & $1610 \%$ \\
\hline
\end{tabular}

Fonte: Os autores (2018)

Verifica-se uma discrepância significativa dos valores referentes aos indicadores absolutos calculados para o processo em estudo frente às metas de idealidade propostas.

\subsection{AVAliaÇÃo do PROCESSO SEgUNDO O MODELO MATEMÁtico}

Analisando-se os resultados obtidos pode-se primeiramente constatar uma grande quantidade de etapas associadas impostas ao consumidor, o que, em uma primeira instancia, já possibilita afirmar que na maior parte do processo o cliente realiza procedimentos que não estão diretamente ligados à sua razão de consumo, e desta forma não lhe agregam valor. Tal fato se reflete no indicador “densidade de etapas de valor” (apenas 18\%).

Dessa forma, um processo alternativo que eliminasse ao máximo tais etapas agregaria maior percentual de valor transmitido ao cliente. Uma consequência direta da observação anterior é a quantidade de desperdício absoluto intrínseco ao processo resultando em um desperdício crítico e urgente (percentual de desperdício absoluto em relação ao tempo total) de $92 \%$. Somando-se os desperdícios gerados pela duração excessiva das etapas fim obtemos e um desperdício emergencial (desperdício total) 95\%. Observa-se que, pelo modelo, tais percentuais foram agravados pela ausência de (medidas) geradoras de valor complementar e/ou subsidiário ao sistema, resultando em um grau de atenuidade nulo. Adicionalmente, obteve-se apenas 5\% de valor real gerado ao cliente. Finalmente verifica-se que a variabilidade do processo como um todo apresenta valores muito elevados (1610\%) representando uma discrepância significativa do padrão estabelecido.

\section{PROPOSTA DE SOLUÇÃO PARA O PROCESSO ATUAL}

Uma vez descrito, vivenciado e analisado o processo de vistoria vigente, o estudo foi focalizado na proposição de melhorias que pudessem efetivamente potencializar o valor revertido ao cliente, tendo como base teórica a filosofia lean.

Para tanto aplicou-se a metodologia do sistema ideal, na qual, partindo-se de um sistema ideal, teórico, onde só houvessem etapas fim (geradoras de valor), acrescentam-se a este, realística e minimamente etapas associadas inevitáveis (geradoras de desperdício).

Em suma, a metodologia propõe uma linha de raciocínio reverso ao usual, ou seja, ao invés de se pensar no estado atual e eliminar os desperdícios vigentes, imagina-se em um estado ideal, sem desperdícios, e acrescentam-se, desperdícios mínimos e inevitáveis. Com isso, procura-se ampliar os horizontes de pensamento, quebrando barreiras impostas pela visão do estado atual e amplificando a possibilidade de propostas mais disruptivas e inovadoras.

Com base nessa linha de pensamento verifica-se que, em um sistema ideal para o caso em estudo, sem nenhum tipo de desperdício, as únicas etapas existentes seriam as 
etapas fim, representadas por:

- Agendamento da vistoria;

- Vistoria;

- Recebimento do novo CRLV;

Considerando este estado teórico, foi idealizada a proposta de melhoria que se segue.

\subsection{Proposta de Vistoria Veicular in loco}

Procurou-se pensar em eliminar a necessidade de deslocamento do usuário e levar a vistoria até sua residência ou local de trabalho (reduzindo, neste caso, a dificuldade de vistoriar o veículo em horários comerciais). Inspirado em serviços de medição in loco como os realizados por agentes das empresas de água, energia elétrica ou seguradoras de veículos - a proposta seria que os vistoriadores realizassem todo o processo de avaliação do veículo na residência do condutor, eliminando para este, desperdícios como deslocamentos, filas, esperas e outros. O processo proposto seria composto teoricamente por 6 simples etapas sendo estas:

- Agendamento da vistoria (etapa fim);

- Recepção do vistoriador (etapa associada);

- Set up (etapa associada);

- Vistoria (etapa fim);

- Atualização do CRLV (etapa fim);

- Despedir-se do vistoriador (etapa associada).

\subsection{REQUISITOS NECESSÁRIOS À PROPOSTA DE VISTORIA VEICULAR IN LOCO}

A proposta apresentada consideraria, além da modificação do processo, alguns requisitos necessários à sua efetiva implantação, considerando tecnologias e organização de pessoal. Um levantamento dos requisitos mínimos necessários, resultou nos seguintes itens:

- Implementação de um CRLV eletrônico;

- Desenvolvimento de sistema de T.I.;

- A utilização de analisadores de emissões veiculares portáteis;

- Recursos humanos necessários às vistorias in loco.

Tecnologias e ferramentas de baixo custo que por si só não imprimiriam ao provedor um peso financeiro elevado e possivelmente reduziriam os custos associados à operação vigente.

\subsection{EMBASAMENTO DA PROPOSTA DE VISTORIA VEICULAR IN LOCO}

\subsubsection{CNH e CRLV Digitais}

Recentemente, o DETRAN RJ lançou a versão digital do Certificado de Registro e Licenciamento de Veículo (CRLV). O órgão aderiu ao aplicativo CDT - Carteira Digital de Trânsito, que reúne em um dispositivo móvel os documentos que são de porte obrigatório para o condutor de veículo - o CRLV e a CNH (que teve sua versão digital lançada em julho de 2018). CRLV digital é disponibilizada mesmo que o proprietário esteja sem acesso à internet, já que o aplicativo gera um arquivo pdf com $Q R$ Code e assinatura digital. A autenticidade do CRLV digital é garantida por esse código, utilizado para verificar se há 
alguma falsificação no documento durante uma fiscalização. Todas as informações do documento impresso estão na versão eletrônica do CRLV, que tem a mesma validade jurídica do CRLV físico.

\subsubsection{Emissão digital do laudo}

O laudo da vistoria poderia ser facilmente emitido para o sistema e para o condutor via email como já fazem correntemente empresas de e-commerce com a nota fiscal eletrônica enviadas para o e-mail ao consumidor.

\subsubsection{Analisadores portáteis de emissões veiculares}

Analisadores portáteis de emissões de gases de combustão são facilmente encontrados no mercado, possibilitando medidas de alta precisão em qualquer lugar, o que viabilizaria a medição in loco dos veículos a serem vistoriados.

\subsection{DETALHAMENTO DO PROCESSO DE VISTORIA VEICULAR IN LOCO}

O processo reformulado seria iniciado, conforme o atual, pelo agendamento da vistoria, selecionando-se o endereço, data e horário para a visita do vistoriador. Feito isso, as próximas etapas se desenvolveriam pela ida do fiscal à residência ou local marcado, onde este seria recepcionado e iniciaria o set up da vistoria, semelhante ao atual. A vistoria sendo realizada e concluída permitiria ao avaliador emitir o laudo eletronicamente (através de um smartphone específico ou palmtop), comunicar o resultado e, em caso de aprovação, atualizar o CRLV digital através do sistema, confirmando assim sua renovação e regularidade.

A figura 2 apresenta um diagrama esquemático do sistema proposto.

Figura 2 - Representação esquemática do processo de vistoria in loco

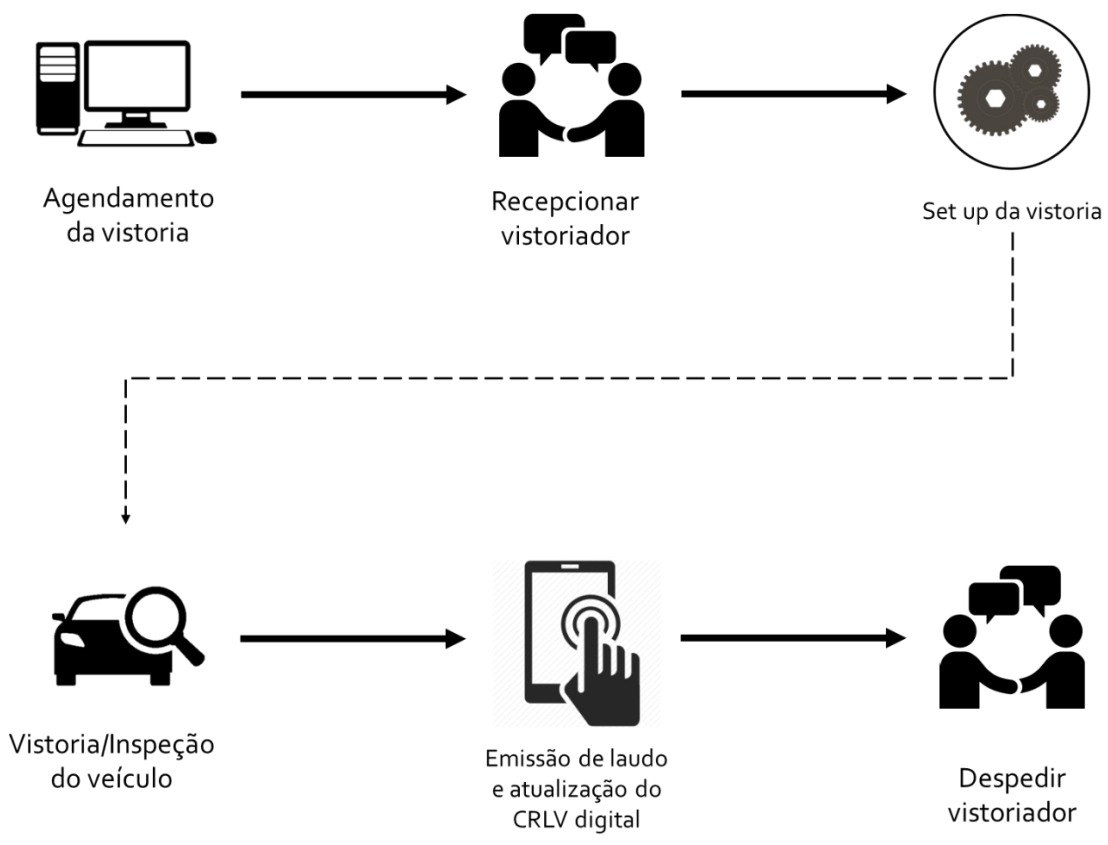

Fonte: Os autores (2018)

O esquema apresentado na figura 2 exibe um processo mais enxuto, eliminando diversas etapas custosas ao consumidor. 


\subsection{AVALIAÇÃo DA PROPOSTA DE VISTORIA VEICULAR IN LOCO}

Após a proposição e estruturação da proposta apresentada são estimados os tempos necessários para a execução de cada uma das etapas componentes e classificadas segundo sua ligação com o objetivo de consumo (etapas fim e etapas associadas).

A tabela 6 apresenta a classificação e os tempos estimados para cada uma das etapas da proposta.

Tabela 6 - Tempos estimados para as etapas da proposta de vistoria in loco

\begin{tabular}{lcc}
\hline \multicolumn{1}{c}{ Etapa } & Classificação & Tempos Tef(i) /Tea(j) \\
\hline Agendamento da vistoria & Etapa fim & $00: 03: 00$ \\
Recepção do vistoriador & Etapa Associada & $00: 02: 00$ \\
Set up & Etapa Associada & $00: 02: 00$ \\
Vistoria & Etapa fim & $00: 03: 00$ \\
Atualização do CRLV & Etapa fim & $00: 01: 00$ \\
Despedir vistoriador & Etapa Associada & $00: 02: 00$ \\
\hline Tempo total estimado para o processo & & $00: 13: 00$ \\
\hline
\end{tabular}

Fonte: Os autores (2018)

Aplicando-se as estimativas ao modelo matemático e calculando-se suas variáveis absolutas e indicadores, obtiveram-se os resultados exibidos respectivamente nas tabelas 7 e 8.

Tabela 7: Indicadores absolutos da proposta vistoria in loco.

\begin{tabular}{lc}
\hline \multicolumn{1}{c}{ Indicadores absolutos } & Valor \\
\hline Quantidade de etapas fins $(\mathrm{m})$ & 3 \\
Quantidade de etapas associadas $(\mathrm{n})$ & 3 \\
Tempo total de etapas fim $\left(\mathrm{T}_{\mathrm{ef}}\right)$ & $00: 07: 00$ \\
Tempo total de etapas associadas $\left(\mathrm{T}_{\text {ea }}\right)$ & $00: 06: 00$ \\
Tempo total de duração do processo de consumo $(\mathrm{T})$ & $00: 13: 00$ \\
Tempo total de valor do consumo $(\mathrm{V})$ & $00: 07: 00$ \\
Tempo total de desperdício das etapas fim - $\left[\mathrm{D}_{\mathrm{ef}}(\mathrm{T})\right]$ & $00: 00: 00$ \\
Tempo total de desperdício das etapas associadas $-\left[\mathrm{D}_{\mathrm{ea}}(\mathrm{T})\right]$ & $00: 06: 00$ \\
Tempo total de desperdício $(\mathrm{D})$ & $00: 06: 00$ \\
Tempo total de valor complementar $\left(\mathrm{V}_{\mathrm{c}}\right)$ & $00: 00: 00$ \\
Tempo total de valor subsidiário $\left(\mathrm{V}_{\mathrm{s}}\right)$ & $00: 00: 00$ \\
\hline
\end{tabular}

Fonte: Os autores (2018)

Considerando os resultados obtidos para o atual processo de vistoria veicular, podese verificar uma redução das etapas pelas quais o consumidor deve passar para completar sua jornada. Esta, que atualmente é composta por 17 etapas, poderia ser potencialmente reduzida para apenas 6. 
Tabela 8: Indicadores relativos da proposta de vistoria in loco.

\begin{tabular}{lcc}
\hline \multicolumn{1}{c}{ Indicadores relativos } & Meta & Valor \\
\hline Valor real & $100 \%$ & $54 \%$ \\
Densidade de etapas de valor & $100 \%$ & $50 \%$ \\
Desperdício crítico e urgente & $0 \%$ & $46 \%$ \\
Desperdício emergencial & $0 \%$ & $46 \%$ \\
Desperdício total & $0 \%$ & $46 \%$ \\
Grau de atenuidade de desperdício & $100 \%^{*}$ & $0 \%$ \\
\hline
\end{tabular}

Fonte: Os autores (2018)

Analisando-se os resultados da tabela 8 verifica-se que o sistema proposto conferiu ao processo um aumento do valor real de 5\% para 54\% (+49\%) estando este fortemente ligado à elevação da densidade das etapas de valor (de 18\% para 50\%) e eliminação de etapas de desperdício.

As tabelas 9 e 10 trazem respectivamente o comparativo dos indicadores absolutos e relativos entre o processo de vistoria convencional e a proposta de vistoria in loco.

Tabela 9: Comparação entre os indicadores absolutos do processo de vistoria veicular convencional e a proposta de vistoria veicula in loco.

\begin{tabular}{lcc}
\hline \multicolumn{1}{c}{ Indicadores absolutos do processo } & Vistoria Convencional & Vistoria in loco \\
\hline Quantidade de etapas fins (m) & 3 & 3 \\
Quantidade de etapas associadas (n) & 14 & 3 \\
Tempo total de etapas fim & $00: 09: 42$ & $00: 07: 00$ \\
Tempo total de etapas associadas & $01: 57: 59$ & $00: 06: 00$ \\
Tempo total de duração do processo & $02: 07: 41$ & $00: 13: 00$ \\
Tempo total de valor do consumo & $00: 06: 54$ & $00: 07: 00$ \\
Tempo total de desperdício das etapas fím & $00: 02: 48$ & $00: 00: 00$ \\
Tempo total de desperdício das etapas associadas & $01: 57: 59$ & $00: 06: 00$ \\
Tempo total de desperdício & $02: 00: 47$ & $00: 06: 00$ \\
\hline
\end{tabular}

Fonte: Os autores (2018)

Percebe-se que, em termos do tempo total despendido pelo usuário seria possível obter uma redução de $2 \mathrm{~h}$ 08min para apenas $13 \mathrm{mim}$, representando uma redução percentual de aproximadamente $90 \%$. 
Tabela 10: Comparação entre os indicadores relativos do processo de vistoria veicular convencional e a proposta de vistoria veicular in loco.

\begin{tabular}{lcc}
\hline \multicolumn{1}{c}{ Indicadores relativos do processo } & Vistoria Convencional & Vistoria in loco \\
\hline Valor real & $5 \%$ & $54 \%$ \\
Densidade de etapas de valor & $18 \%$ & $50 \%$ \\
Desperdício crítico e urgente & $92 \%$ & $46 \%$ \\
Desperdício emergencial & $95 \%$ & $46 \%$ \\
Desperdício total & $95 \%$ & $46 \%$ \\
Grau de atenuidade de desperdício & $0 \%$ & $0 \%$ \\
\hline
\end{tabular}

Fonte: Os autores (2018)

Avaliando os desperdícios gerados, a proposta de reformulação possibilita uma redução teórica do desperdício total de 95\% para 46\% (-49\%). Adicionalmente, acredita-se que, pela eliminação de deslocamentos, filas, esperas e outros, sua experiencia em relação ao processo lhe conferiria um sentimento positivo.

\section{CONSIDERAÇÕES FINAIS}

Diante do objetivo proposto de analisar um processo de consumo presente no setor público através da modelagem matemática apresentada, e com isso, propor melhorias ao estado atual, observou-se que a utilização dos princípios da filosofia lean aliada ao modelo matemático, possibilitou reformular a jornada de consumo referente à vistoria veicular do DETRAN RJ, de forma a reduzir o número total de etapas em $64,7 \%$, os tempos não agregadores de valor em 95\%, além propiciar 54\% de tempo agregador de valor frente aos $7,81 \%$ presentes no processo vigente.

O estudo evidencia que a metodologia utilizada pode ser aplicada amplamente em outros processos de consumo, possibilitando uma análise quantitativa detalhada dos valores e desperdícios gerados aos clientes, apresentando com isso um potencial de melhoria significativo para os consumidores e para o nível de serviço de inúmeras organizações, tanto públicas quanto privadas.

\section{REFERÊNCIAS BIBLIOGRÁFICAS}

[1] COSTA, R.S. e JARDIM, E.G.M. Produção e Serviços: reflexões e conceitos. São Paulo: Atlas, 2015.

[2] GUIMARÃES, L.A. e GUIMARÃES, C.R. Utilização da construção enxuta no planejamento e controle de obras na construção civil. João Pessoa: XXXVI Encontro Nacional de Engenharia de Produção (ENEGEP), 2016. 
[3] JARDIM, E. G. M. Passos para aplicação do modelo matemático para análise do fluxo de valor de um processo de consumo. Notas de aula da disciplina tópicos especiais em sistemas de informação - Rio de Janeiro: UFRJ, 2014.

[4] WOMACK, James P.; JONES, Daniel T. Soluções Enxutas: Como Empresas e Clientes Conseguem Juntos Criar Valor e Riqueza. Editora Campus, 2006.

[5] http://www.detran.rj.gov.br/_monta_aplicacoes.asp?doc=10767\&cod=14\&tipo=exibe_noticias\&pag_noticias=true Acessado em 17/05/2019 